\title{
Normal and Dirac fermions in graphene multilayers: Tight-binding description of the electronic structure
}

\author{
B. Partoens* and F. M. Peeters ${ }^{\dagger}$ \\ Departement Fysica, Universiteit Antwerpen, Groenenborgerlaan 171, B-2020 Antwerpen, Belgium \\ (Received 5 February 2007; published 8 May 2007)
}

\begin{abstract}
Within a tight-binding approach we show that around the $K$ point of the energy spectrum Dirac fermions are present in $A B$ stacked graphene multilayers if they have mirror plane symmetry. In other words, Dirac fermions are present in graphene stacks with an odd number of layers. For an even number of stacked graphene layers, only normal fermions with a parabolic energy dispersion are found near the $K$ point.
\end{abstract}

DOI: 10.1103/PhysRevB.75.193402

Recently, there has been an increased amount of experimental and theoretical interest in the electronic properties of ultrathin graphite films. Based on an analysis of the quantum Hall effect and Shubnikov de Haas oscillations in a single graphene layer it was concluded that around the Fermi energy the carriers are Dirac fermions with a linear energy spectrum. ${ }^{1,2}$ Remarkably, in quasi-two-dimensional (2D) highly oriented pyrolytic graphite (HOPG) both conventional electrons with parabolic type of energy spectrum and Diraclike electrons with linear spectrum were observed. This was based on quantum de Haas-van Alphen and Shubnikov-de Haas oscillations ${ }^{3}$ and the quantum Hall effect ${ }^{4}$ in bulk HOPG samples, and angle resolved photoemission spectroscopy experiments on $A B$-stacked graphite, ${ }^{5}$ which proved the coexistence of Dirac-like holes and normal (massive) electrons located at $H$ and $K$ points of the Brillouin zone, respectively.

These results suggest that Dirac fermions should also be present in other multilayer structures of graphene. But in Refs. 6 and 7 it was shown that Dirac fermions are not
PACS number(s): 81.05.Uw, 73.63.Bd, 73.43.Cd

present in bilayer graphene, indicating that the story is much more complicated. Previous theoretical works ${ }^{8-11}$ already indicated that charge carriers in bilayer graphene exhibit a parabolic spectrum. On the other hand, in Ref. 12 a clear Dirac nature of the charge carriers in ultrathin epitaxial graphene systems was observed from magnetoresistance and quantum Hall measurements, and angle resolved photoemission spectroscopy experiments. The authors of Ref. 12 claimed that this observation is due to the response of the electrons in the single graphene layer at the graphite-SiC substrate interface.

However, in this work we will show that Dirac fermions are present in all $A B$ stacked graphene multilayers consisting of an odd number of layers. Our proof is based on a tightbinding description of the electronic structure. The latter was used earlier to investigate the evolution of the electronic structure around the $K$ point when we go from a single graphene layer to bulk graphite. ${ }^{9}$

As shown in Ref. 9, the tight-binding Hamiltonian for an arbitrary number of $A B$ stacked graphene layers is given by

$$
\left(\begin{array}{cc|cc|cc|cc|l}
\Delta+\gamma_{5} & \gamma_{0} f & \gamma_{1} & -\gamma_{4} f^{*} & \gamma_{5} / 2 & 0 & 0 & 0 & \cdots \\
\gamma_{0} f^{*} & \gamma_{2} & -\gamma_{4} f^{*} & \gamma_{3} f & 0 & \gamma_{2} / 2 & 0 & 0 & \cdots \\
\hline \gamma_{1} & -\gamma_{4} f & \Delta+\gamma_{5} & \gamma_{0} f^{*} & \gamma_{1} & -\gamma_{4} f & \gamma_{5} / 2 & 0 & \cdots \\
-\gamma_{4} f & \gamma_{3} f^{*} & \gamma_{0} f & \gamma_{2} & -\gamma_{4} f & \gamma_{3} f^{*} & 0 & \gamma_{2} / 2 & \cdots \\
\hline \gamma_{5} / 2 & 0 & \gamma_{1} & -\gamma_{4} f^{*} & \Delta+\gamma_{5} & \gamma_{0} f & \gamma_{1} & -\gamma_{4} f^{*} & \cdots \\
0 & \gamma_{2} / 2 & -\gamma_{4} f^{*} & \gamma_{3} f & \gamma_{0} f^{*} & \gamma_{2} & -\gamma_{4} f^{*} & \gamma_{3} f & \cdots \\
\hline 0 & 0 & \gamma_{5} / 2 & 0 & \gamma_{1} & -\gamma_{4} f & \Delta+\gamma_{5} & \gamma_{0} f^{*} & \cdots \\
0 & 0 & 0 & \gamma_{2} / 2 & -\gamma_{4} f & \gamma_{3} f^{*} & \gamma_{0} f & \gamma_{2} & \cdots \\
\hline \vdots & \vdots & \vdots & \vdots & \vdots & \vdots & \vdots & \vdots & \ddots
\end{array}\right),
$$

where the rows and columns are ordered according to atom $A$ from layer 1, atom $B$ from layer 1, atom $A$ from layer 2, atom $B$ from layer 2, etc. In Eq. (1) $\gamma_{0}, \gamma_{1}, \gamma_{2}, \gamma_{3}, \gamma_{4}, \gamma_{5}$, and $\Delta$ are the Slonczewski-Weiss-McClure parameters and the function $f$ stands for

$$
f\left(k_{x}, k_{y}\right)=e^{i k_{x} a / \sqrt{3}}+2 e^{-i k_{x} a / 2 \sqrt{3}} \cos k_{y} a / 2,
$$

with $a$ the length of the in-plane lattice vector. The six parameters $\gamma_{0}, \gamma_{1}, \gamma_{2}, \gamma_{3}, \gamma_{4}$, and $\gamma_{5}$ express the couplings between the different atoms (for details see Ref. 9).

After diagonalizing the Hamiltonian (1) we obtain the energy spectrum. In the following, we limit ourselves to the important region of the spectrum situated around the $K$ point. This narrow region of the spectrum is shown in Fig. 1 for $N=1$ up to 10 layers for wave vectors in the direction of the $\Gamma$ and $M$ point. Notice that only for an odd number of layers, a linear spectrum exists around the Fermi energy. Thus only systems which have a mirror inversion plane exhibit Dirac 

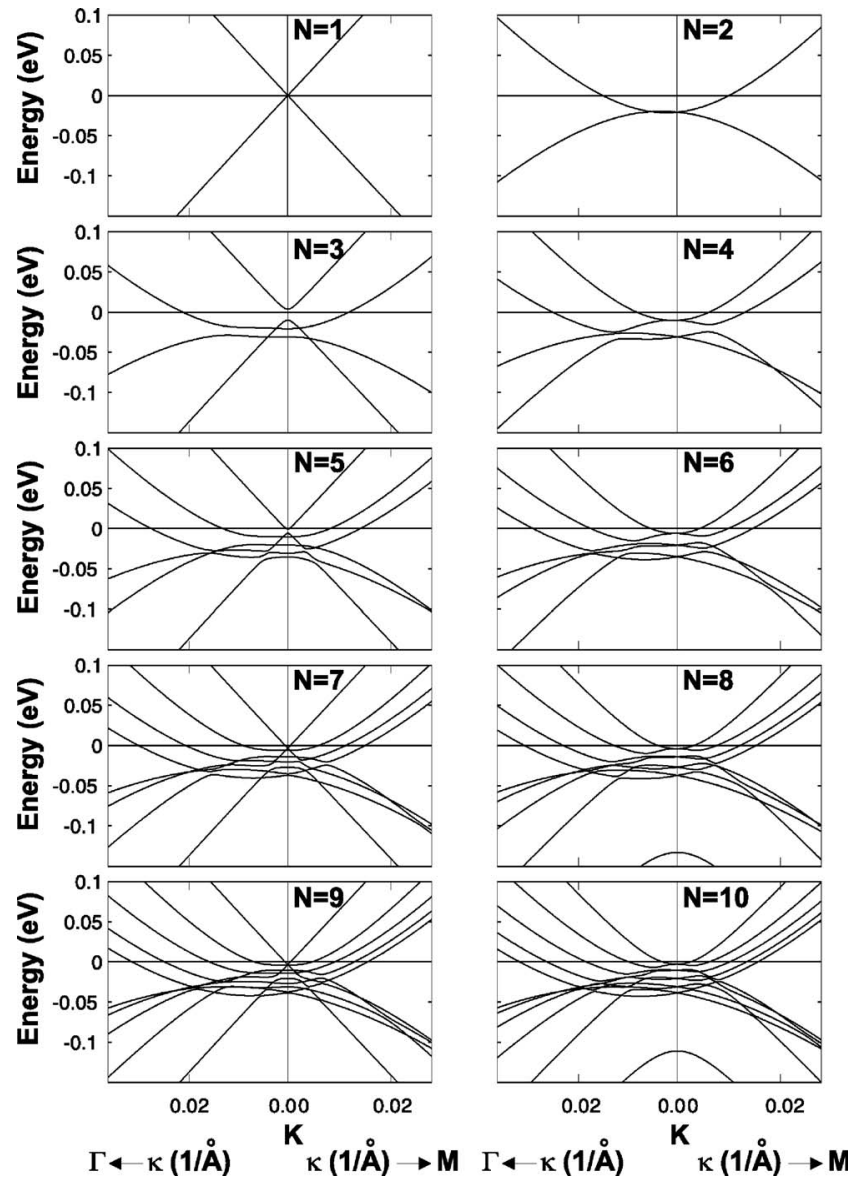

FIG. 1. The band structure of $N$ graphene layers around the $K$ point and the Fermi energy in the direction of the $\Gamma$ point and the $M$ point, for $N=1$ up to 10 .

type charge carriers. Note that the effective speed of light of these Dirac fermions is the same in all these systems (i.e., $\left.\sqrt{3} \gamma_{0} a / 2 \hbar \approx 1.01 \times 10^{6} \mathrm{~m} / \mathrm{s}\right)$. For $2 N$ and $2 N+1 A B$-stacked layers (and $N>1$ ) there are at the Fermi energy $2 N$ parabolic bands around the $K$ point, of which $N$ are electronlike and $N$ holelike bands. The systems with an odd number of layers have additionally a linear energy band. Furthermore, close to the $K$ point crossings and anticrossings occur.
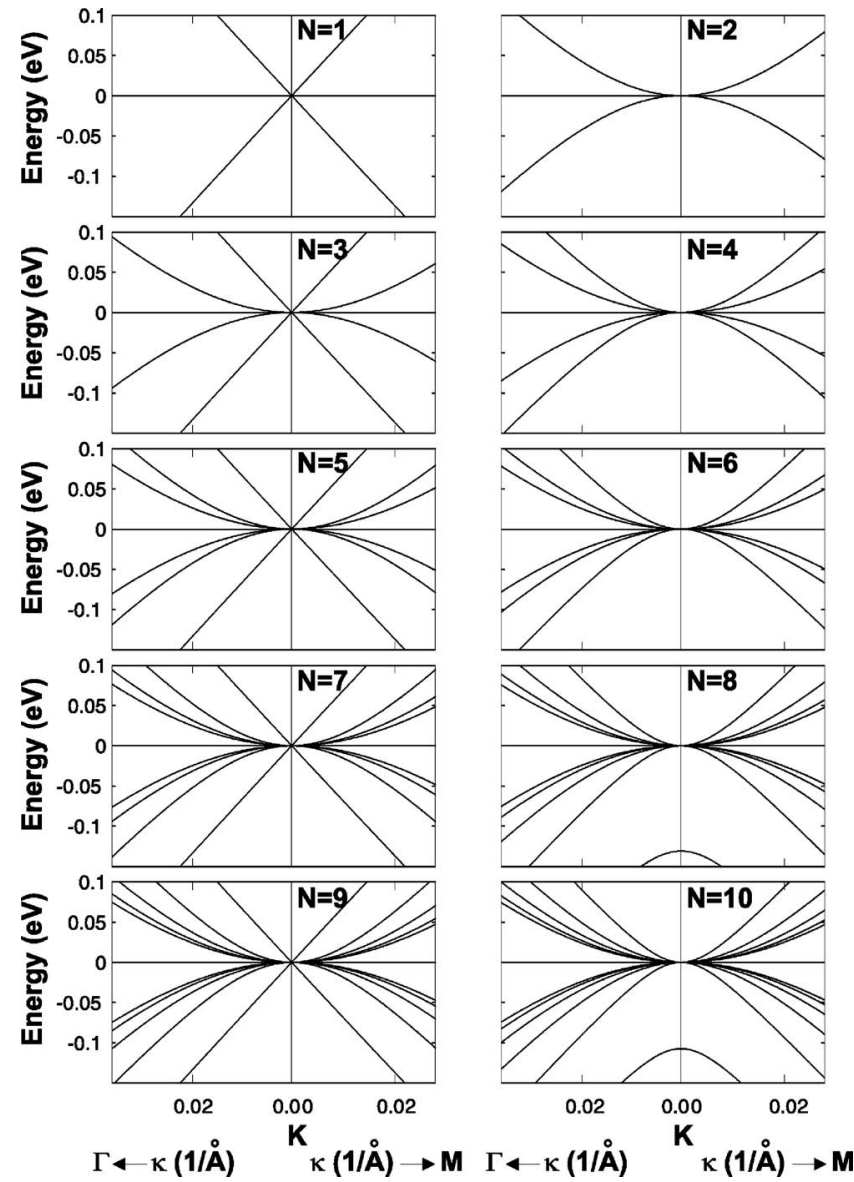

FIG. 2. The same as in Fig. 1 but with $\gamma_{2}=\gamma_{3}=\gamma_{4}=\gamma_{5}=\Delta=0$.

From the form of the Hamiltonian it is not directly apparent how to extract the linear energy band out of it in case of an odd number of layers. Therefore, let us simplify Eq. (1) such that the number of bands and the appearance of a linear band for an odd number of layers can be recovered. If we neglect all interactions except between the nearest neighbor atoms in the same layer and between $A$-type atoms between adjacent layers (which are on top of each other), i.e., we put $\gamma_{2}=\gamma_{3}=\gamma_{4}=\gamma_{5}=\Delta=0$, the Hamiltonian (1) reduces to

$$
\left(\begin{array}{cc|cc|cc|cc|c}
0 & \gamma_{0} f & \gamma_{1} & 0 & 0 & 0 & 0 & 0 & \cdots \\
\gamma_{0} f^{*} & 0 & 0 & 0 & 0 & 0 & 0 & 0 & \cdots \\
\hline \gamma_{1} & 0 & 0 & \gamma_{0} f^{*} & \gamma_{1} & 0 & 0 & 0 & \cdots \\
0 & 0 & \gamma_{0} f & 0 & 0 & 0 & 0 & 0 & \cdots \\
\hline 0 & 0 & \gamma_{1} & 0 & 0 & \gamma_{0} f & \gamma_{1} & 0 & \cdots \\
0 & 0 & 0 & 0 & \gamma_{0} f^{*} & 0 & 0 & 0 & \cdots \\
\hline 0 & 0 & 0 & 0 & \gamma_{1} & 0 & 0 & \gamma_{0} f^{*} & \cdots \\
0 & 0 & 0 & 0 & 0 & 0 & \gamma_{0} f & 0 & \cdots \\
\hline \vdots & \vdots & \vdots & \vdots & \vdots & \vdots & \vdots & \vdots & \ddots
\end{array}\right) .
$$


The corresponding energy spectrum around the $K$ point for 1 up to $N$ layers is given in Fig. 2. Notice that we recover many of the features of the original tight-binding spectrum (Fig. 1) except for the shifts and anticrossings of the bands. The linear spectrum is indeed only present for an odd number of layers. The above Hamiltonian allows for a simple analytical expression for its characteristic polynomial, of which the zeros determine the energy spectrum. We obtain a recursive relation for the characteristic polynomial $C P(N)$ for $N$ graphene layers, which is given by

$$
C P(N)=C P(1) C P(N-1)-E^{2} \gamma_{1}^{2} C P(N-2),
$$

with $C P(0)=1$ and $C P(1)=E^{2}-\gamma_{0}^{2}\left|f\left(k_{x}, k_{y}\right)\right|^{2}$. The latter characteristic polynomial for a single graphene layer is responsible for the linear spectrum around the $K$ point. Indeed, by requesting $C P(1)=0$ we obtain the linear spectrum $E$ $= \pm(\sqrt{3} / 2) \gamma_{0} \kappa$ up to first order in $\kappa$ which is the distance of the wave vector from the $K$ point. From the recursive relation (4) it is apparent that only for an odd number of layers $C P(1)$ can be factored out:

$$
C P(2 N+1)=C P(1) \sum_{i=0}^{N}\left(-E \gamma_{1}^{2}\right)^{i} C P[2(N-i)]
$$

while for an even number of layers we have

$$
C P(2 N)=C P(1) \sum_{i=0}^{N-1}\left(-E \gamma_{1}^{2}\right)^{i} C P[2(N-i)-1]+\left(-E^{2} \gamma_{1}^{2}\right)^{N}
$$

As a consequence, only for an odd number of graphene layers, Dirac fermions are present. This result can also be deduced from a general dispersion relation for a system of $N$ graphene layers which was obtained in Ref. 11 within the same approximation that hopping between planes takes place only between atoms which are on top of each other in neighboring planes.

In conclusion, Dirac fermions are not only present in a single graphene layer ${ }^{1,2}$ but also in $A B$-stacked graphite as recently observed experimentally in Refs. $3-5$. The Dirac fermions in graphite have a hole character due to the position of the Fermi energy and are situated around the $H$ point. However, we showed that in stacks of graphene layers these Dirac charge carriers are only present for an odd number of layers, and thus in systems with a mirror plane. In stacked graphene layers, these Dirac fermions are located around the $K$ point. The multiband structure appearing at the $K$ point as a consequence of the different layers of graphene can be viewed as the discrete projection of the energy spectrum along the $K$ - $H$ direction of graphite.

This work was supported by the Flemish Science Foundation (FWO-Vl), the "Belgian Science Policy," and BOFTOP (University of Antwerp). We thank A. Geim for stimulating correspondence.
*Electronic address: bart.partoens@ua.ac.be

${ }^{\dagger}$ Electronic address: francois.peeters@ua.ac.be

${ }^{1}$ K. S. Novoselov, A. K. Geim, S. V. Morozov, D. Jiang, M. I. Katsnelson, I. V. Grigorieva, S. V. Dubonos, and A. A. Firsov, Nature (London) 438, 197 (2005).

${ }^{2}$ Y. Zhang, Y.-W. Tan, H. L. Stormer, and P. Kim, Nature (London) 438, 201 (2005).

${ }^{3}$ I. A. Luk'yanchuk and Y. Kopelevich, Phys. Rev. Lett. 93, 166402 (2004).

${ }^{4}$ I. A. Luk'yanchuk and Y. Kopelevich, Phys. Rev. Lett. 97, 256801 (2006).

${ }^{5}$ S. Y. Zhou, G.-H. Gweon, J. Graf, A. V. Fedorov, C. D. Spataru, R. D. Diehl, Y. Kopelevich, D.-H. Lee, Steven G. Louie, and A. Lanzara, Nat. Phys. 2, 595 (2006).
${ }^{6}$ K. S. Novoselov, E. McCann, S. V. Morozov, V. I. Fal'ko, M. I. Katsnelson, U. Zeitler, D. Jiang, F. Schedin, and A. K. Geim, Nat. Phys. 2, 177 (2006).

${ }^{7}$ T. Ohta, A. Bostwick, T. Seyller, K. Horn, and E. Rotenberg, Science 313, 951 (2006).

${ }^{8}$ E. McCann and V. I. Fal'ko, Phys. Rev. Lett. 96, 086805 (2006).

${ }^{9}$ B. Partoens and F. M. Peeters, Phys. Rev. B 74, 075404 (2006).

${ }^{10}$ S. Latil and L. Henrard, Phys. Rev. Lett. 97, 036803 (2006).

${ }^{11}$ F. Guinea, A. H. Castro Neto, and N. M. R. Peres, Phys. Rev. B 73, 245426 (2006).

${ }^{12}$ C. Berger, Z. Song, X. Li, X. Wu, N. Brown, C. Naud, D. Mayou, T. Li, J. Hass, A. N. Marchenkov, E. H. Conrad, Ph. N. First, and W. A. de Heer, Science 312, 1191 (2006). 